\title{
PERFIL POPULACIONAL DE PACIENTES COM DISTÚRBIOS DA COMUNICAÇÃO HUMANA DECORRENTES DE LESÃO CEREBRAL, ASSISTIDOS EM HOSPITAL TERCIÁRIO
}

\author{
Populational profile of patients with human communication \\ disorders after brain injury, assisted in a tertiary hospital
}

\author{
Thais Rodrigues Talarico ${ }^{(1)}$, Mayra Jacuviske Venegas ${ }^{(2)}$, Karin Zazo Ortiz ${ }^{(3)}$
}

\begin{abstract}
RESUMO
Objetivo: caracterizar a população de pacientes com lesão neurológica, queixa e presença de distúrbio da comunicação, assistidos num Hospital Terciário. Os objetivos específicos foram: verificar a prevalência de cada tipo de transtorno de fala e/ou de linguagem, da etiologia neurológica e a origem dos encaminhamentos em relação ao total de pacientes. Método: Trata-se de um estudo retrospectivo, em que foram analisados todos os prontuários dos pacientes atendidos em 5 anos (2002 a 2006), no Núcleo de Investigação e Intervenção Fonoaudiológica em Neuropsicolingüística do Departamento de Fonoaudiologia da Universidade Federal de São Paulo,. Resultados: o estudo revelou 244 casos atendidos entre 2002 e 2006. Quanto à caracterização da amostra encontramos: quanto ao sexo, $56 \%$ eram homens; quanto à idade, 44,3\% encontraram-se entre 41- 64 anos; quanto à escolaridade, $39,8 \%$ dos pacientes apresentaram 1 a 4 anos de escolaridade; quanto aos encaminhamentos para o atendimento no setor $35,7 \%$ eram procedentes do ambulatório de Neurologia da UNIFESP; quanto à etiologia da lesão, 69,3\% foram diagnosticados com Acidente Vascular Cerebral (AVC). Sobre o diagnóstico fonoaudiológico, 56,1\% foram diagnosticados com Afasia em relação à linguagem e 33\% com Disartria em relação à fala. Conclusão: a afasia foi o transtorno de linguagem mais prevalente; o mesmo ocorreu com a disartria em relação à fala; o AVC foi a etiologia neurológica predominante; em relação ao número geral de casos avaliado verificou-se que $50 \%$ do grupo atendido estavam em programas de reabilitação; a maioria dos pacientes foram encaminhados pelo serviço de Neurologia do Hospital São Paulo - UNIFESP.
\end{abstract}

Descritores: Transtornos da Comunicação; Traumatismos Encefálicos; Epidemiologia

\section{INTRODUÇÃO}

Os processos de produção da fala e da linguagem englobam atividades distintas do córtex cerebral. Assim, diferentes tipos de alteração no Sistema Nervoso Central (SNC) podem resultar em diversos tipos de distúrbios de linguagem e/ou fala.

(1) Fonoaudióloga formada pela Universidade Federal de São Paulo - UNIFESP, São Paulo, SP, Brasil.

(2) Fonoaudióloga formada pela Universidade Federal de São Paulo - UNIFESP, São Paulo, SP, Brasil.

(3) Fonoaudióloga; Professor Adjunto Doutor do Departamento de Fonoaudiologia da Universidade Federal de São Paulo - UNIFESP, São Paulo, SP, Brasil; Pós-doutorado em Neurociências pela UNIFESP.

Conflito de interesses: inexistente
O Acidente Vascular Cerebral (AVC) e o Traumatismo Cranioencefálico (TCE) são as causas mais frequentes, na prática clínica, para os danos cerebrais. As lesões cerebrais podem resultar em alterações de linguagem e/ou fala. A afasia, geralmente decorrente de uma lesão focal, é um dos distúrbios de linguagem mais comuns. Como alterações de fala frequentemente encontradas podemos considerar as apraxias e disartrias.

Em nosso meio, são escassas as pesquisas que apontem para o diagnóstico neurológico e o de linguagem mais prevalentes na população. A cada 100.000 pacientes que apresentam AVC isquêmico, 43 podem apresentar afasia neste primeiro insulto ${ }^{1}$. O AVC é uma doença comum e de grande impacto na saúde pública e constitui a segunda causa de 
morte em adultos. Como principais complicações tardias poderíamos citar déficits motores, incapacidades funcionais e distúrbios de linguagem² ${ }^{2}$.

Em relação aos TCE's, pesquisas apontam que é a principal causa de morte em pessoas entre 1 e 44 anos. A recuperação dos sobreviventes é marcada por sequelas neurológicas graves e por uma qualidade de vida muito prejudicada. ${ }^{3}$

Entre as principais alterações da comunicação que têm como causa um distúrbio ou dano neurológico estão as afasias, as disartrias e as apraxias. A afasia é uma alteração no conteúdo, na forma e no uso da linguagem e de seus processos cognitivos subjacentes, tais como percepção e memória ${ }^{4}$ As afasias podem ser classificadas em emissivas como as de Broca, de Condução e Transcortical Motora; receptivas como as de Wernicke, Transcortical Sensorial e Amnéstica/Anômica e as formas mistas tais como, Afasia Transcortical Mista, Afasia Mista e a Afasia Motora Mista. Além das alterações de comunicação este acometimento gera impacto na vida social dos pacientes, destacando-se a diminuição da probabilidade de retorno para as atividades profissionais ${ }^{5}$.

Em relação às disartrias, podem ser caracterizadas por disrupturas primárias na articulação e na prosódia ${ }^{6}$. Essas alterações de fala podem influenciar a vida profissional e pessoal do paciente. As disartrias são distúrbios de fala que decorrem de lesão do Sistema Nervoso Central ou Periférico ${ }^{7}$.

Já a apraxia seria a incapacidade de realizar uma ação, movimento ou sequência de movimento. Em relação às apraxias que interferem na comunicação temos a apraxia verbal (ou de fala) e a não verbal. As lesões centrais localizadas nas áreas responsáveis pela sequencialização dos comandos motores da fala são as maiores causadoras desta alteração ${ }^{7}$.

As lesões neurológicas ainda podem causar outros distúrbios comprometendo direta ou indiretamente a comunicação, que ocorrem em menor frequência na população, como as Agnosias, as Dislexias e as Agrafias adquiridas, distúrbios linguísticos - cognitivos entre outras.

Os métodos mais recentes de investigação clínica (neuro-imagem estrutural e funcional), bem como os avanços teóricos nos fazem refletir sobre a concepção das relações lesão-sintoma em relação à linguagem e à fala. Assim, ao se considerar os circuitos neuronais responsáveis pela linguagem e fala, a lesão em uma das partes destes circuitos tenderá a afetar a função como um todo embora em graus e formas variadas ${ }^{8}$.

Em nosso meio são escassas as pesquisas que apontem para a prevalência populacional dos distúrbios da comunicação de origem neurológica bem como a assistência oferecida a estes pacientes quando são consideradas variáveis como tipo de avaliação, tempo médio no serviço e/ou reavaliação, melhoras observadas e/ou terapia, orientação, encaminhamentos, entre outros.

Também não possuímos um estudo que revele qual o número de novos casos surgidos numa determinada população e num determinado intervalo de tempo - o que poderia auxiliar na caracterização da incidência desses distúrbios.

Considerando que há uma vasta possibilidade de diferentes distúrbios da comunicação de origem neurológica, é interessante que conheçamos o perfil das pessoas que necessitam deste atendimento como forma de promover melhor assistência à população.

Assim este estudo tem como objetivo caracterizar a população de pacientes com lesão neurológica com queixa de alteração e presença de distúrbio da comunicação, assistida em um Hospital Terciário. e constou dos seguintes objetivos específicos:

1. Verificar a prevalência de cada tipo de transtorno de fala e/ou de linguagem em relação ao número total de casos atendidos;

2. Verificar a prevalência da etiologia neurológica em relação ao número total de casos atendidos;

3. Verificar a tendência de crescimento dos casos atendidos ano a ano, nos últimos 5 anos; em relação ao tipo de distúrbio de comunicação e em relação à etiologia;

4. Verificar a porcentagem de casos que puderam ser atendidos em programas de reabilitação em relação ao número geral de casos atendidos;

5. Verificar a origem dos encaminhamentos para o atendimento específico, quanto ao local de origem do encaminhamento.

\section{MÉTODO}

Para a realização da presente pesquisa analisamos os prontuários dos pacientes atendidos nos últimos 5 anos (2002 a 2006), por meio de um estudo retrospectivo. Após a primeira análise destes prontuários, os mesmos foram separados por ano, para verificação da prevalência anual de:

a) Dados individuais: sexo, idade, escolaridade;

b) Dados neurológicos: etiologia da lesão

c) Assistência dada ao paciente: avaliação, lista de espera para atendimento, orientação e encaminhamentos feitos para outro serviço;

d) Quanto à assistência fornecida: tempo de permanência em terapia fonoaudiológica e em lista de espera para atendimento;

e) Dados de acompanhamento clínico: paciente encaminhado por outro serviço de outra instituição, ou por outro serviço da UNIFESP; 
encaminhamentos feitos por qual especialidade da área da saúde.

O Ambulatório de Distúrbios Neurológicos Adquiridos da Disciplina de Distúrbios da Comunicação Humana do Departamento de Fonoaudiologia da Universidade Federal de São Paulo Escola Paulista de Medicina está vinculado ao Hospital São Paulo. No serviço atuam fonoaudiólogos que recebem pacientes tanto do próprio hospital como de outros serviços (particulares e públicos). Os mesmos realizam avaliação dos pacientes recebidos. Na última sessão é explicado para o paciente o que foi possível constatar durante a avaliação, o diagnóstico fonoaudiológico e a conduta a ser tomada. O ambulatório pode encaminhar esses pacientes: para um serviço próximo a sua residência, para uma avaliação neurológica mais detalha no próprio HSP, para a lista de espera de terapia fonoaudiológica e/ou o paciente pode ser orientado quanto a sua queixa. Em alguns períodos do ano é realizada triagem para os pacientes que estão na fila de espera para avaliação com o intuito de encaminhar para outro serviço os pacientes que eventualmente estejam esperando por avaliação, mas que não sejam casos de distúrbios da comunicação de origem neurológica.

Esta pesquisa foi aprovada pelo Comitê de Ética da Instituição, Universidade Federal de São Paulo, CEP número 1606/07

A análise descritiva dos dados coletados será apresentada após a tabulação de todos os dados de interesse deste estudo, os mesmos foram submetidos a tratamento estatístico. Todos os gráficos foram construídos segundo o pacote estatístico SPSS (Statistical Pakage for the Social Science) 11.5.1 para Windows.

\section{RESULTADOS}

O número total de atendimentos realizados no Ambulatório de Distúrbios Neurológicos Adquiridos do Departamento de Fonoaudiologia da UNIFESPEPM período de 2002 a 2006 foi de 244 casos. Deste total apenas um sujeito não tinha alteração fonoaudiológica.

\section{Caracterização da Amostra}

Quarenta e quatro por cento dos pacientes eram mulheres.

A classificação por idades está representada na Figura 1.

A escolaridade variou conforme a Figura 2. $\mathrm{Na}$ figura 3 , observamos a distribuição do número de pacientes em relação à procedência do encaminhamento.

$\mathrm{Na}$ Tabela 1, apresentamos as frequências dos diagnósticos fonoaudiológicos realizados no período de 2002 a 2006. Ressaltamos que 99 pacientes tinham mais que um diagnóstico fonoaudiológico, e em 32 casos, não conseguimos concluir o diagnóstico. Esses pacientes foram aqueles que foram atendidos em triagem e foram encaminhados para outros serviços ou que abandonaram o processo de avaliação.

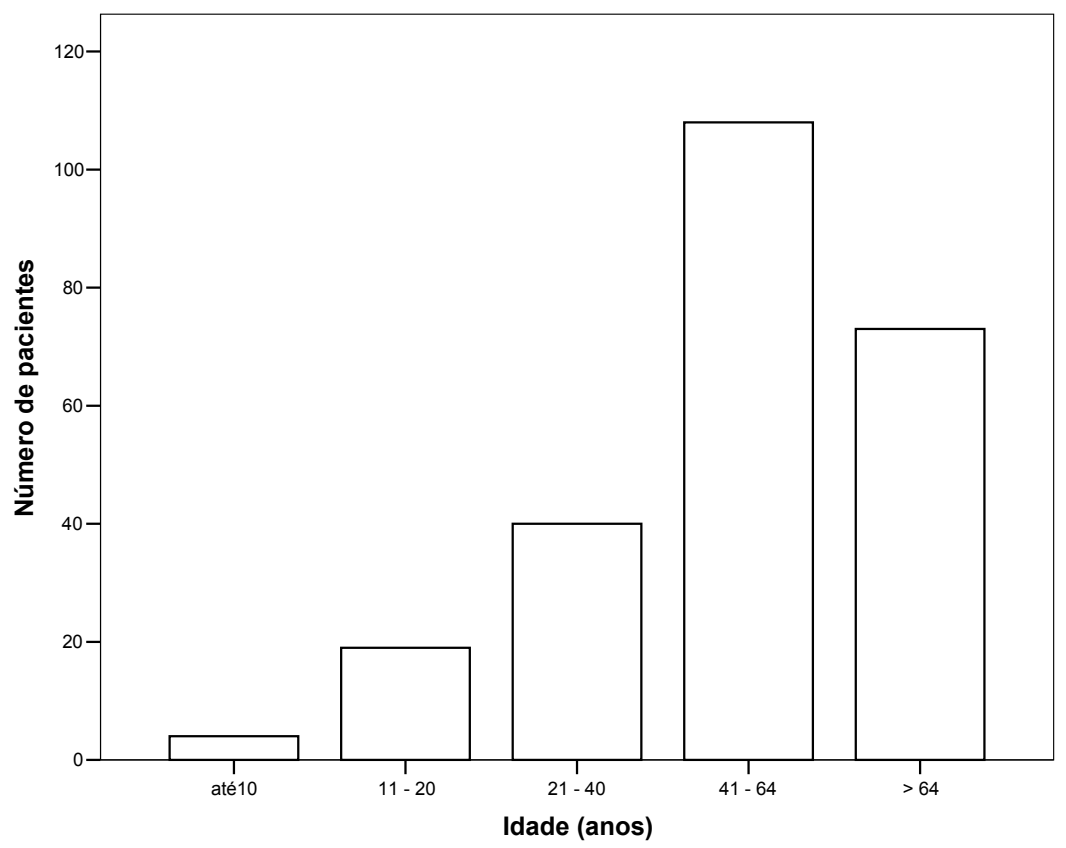

Figura 1 - Distribuição do número total de pacientes em relação à faixa etária. 


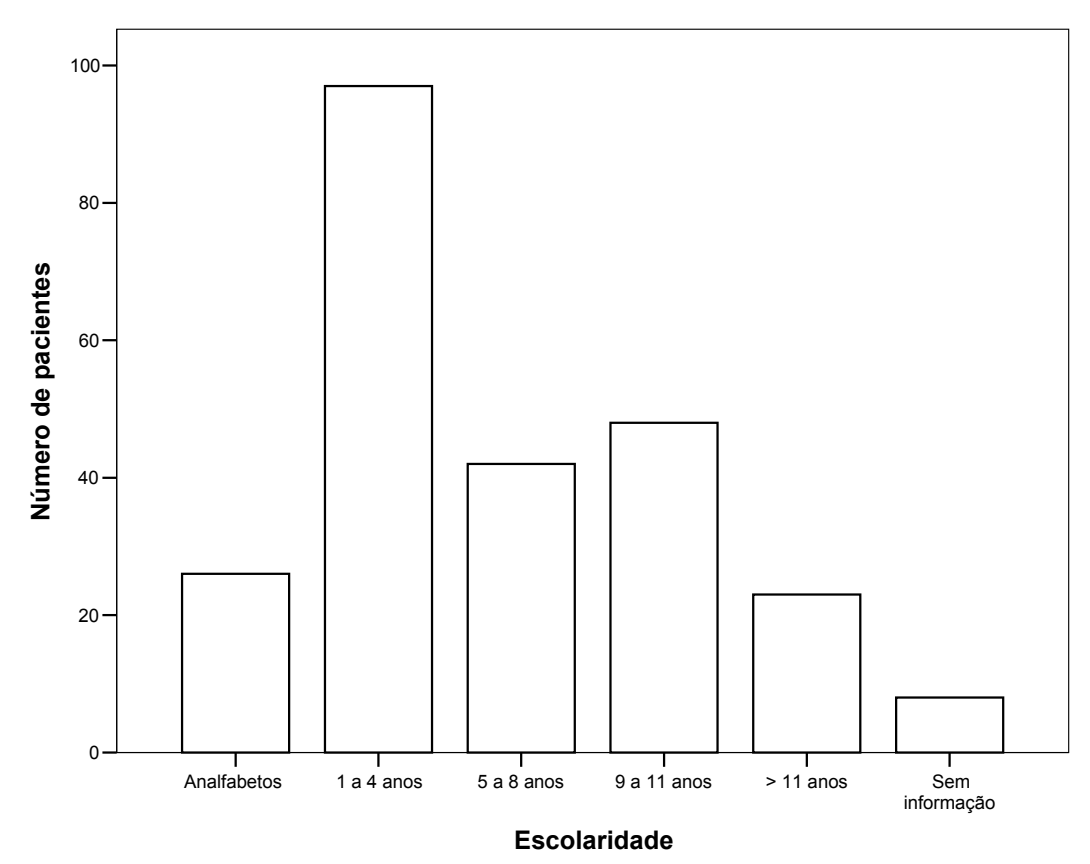

Figura 2 - Distribuição do número total de pacientes em relação à faixa de escolaridade.

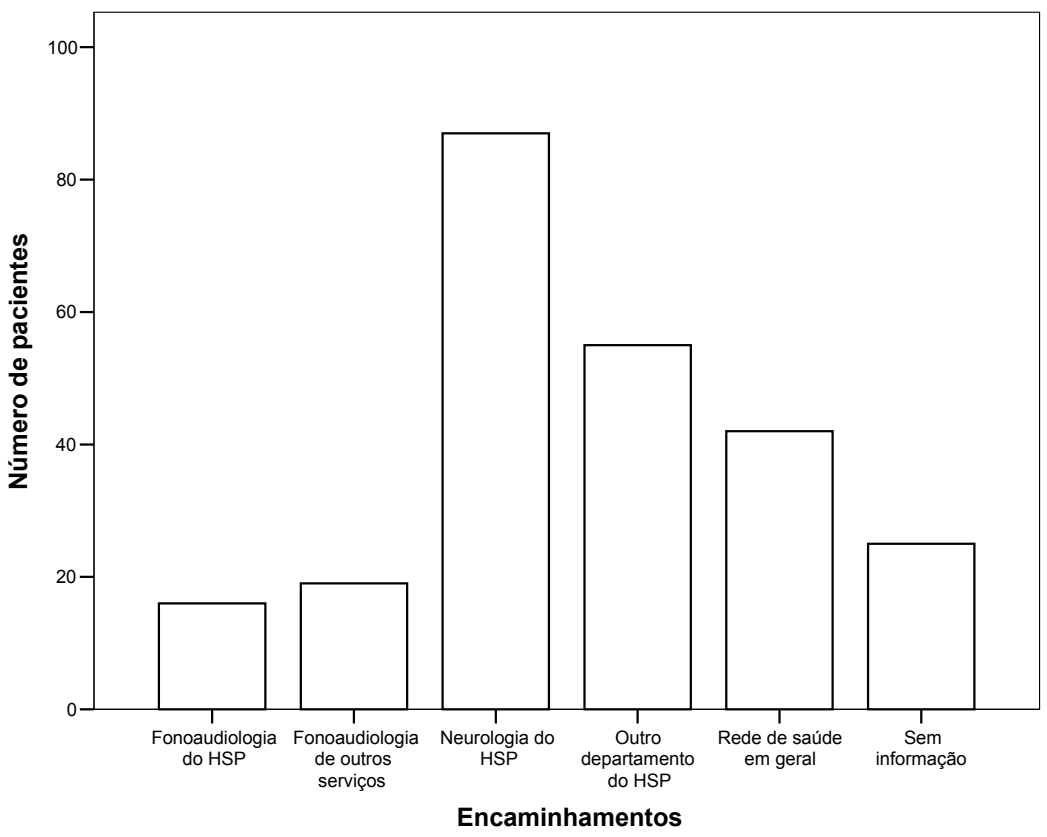

Figura 3 - Distribuição do número total de pacientes em relação à procedência do encaminhamento.

As frequências das etiologias das lesões variaram conforme explicitado na Figura 4.

A Figura 5 apresenta a condição atual dos pacientes atendidos no ambulatório de distúrbios neurológicos adquiridos da UNIFESP de 2002 a 2006.
A Figura 6 representa o tempo (em meses) de terapia realizado pelos pacientes que foram submetidos ao tratamento. Para esta tabela, consideramos apenas os 69 pacientes que realizaram terapia. 
Tabela 1 - Freqüência dos diagnósticos fonoaudiológicos

\begin{tabular}{lcc}
\hline Diagnósticos fonoaudiológicos & Freqüência & Porcentagem \\
\hline Afasia & 119 & 56,1 \\
Dislexia & 2 & 0,9 \\
Agrafia adquirida & 6 & 2,8 \\
Distúrbio sutil de linguagem & 25 & 11,8 \\
Distúrbio lingüístico cognitivo & 20 & 9,4 \\
Distúrbio de aquisição de fala e linguagem & 4 & 1,9 \\
Disartria & 70 & 33 \\
Apraxia & 41 & 19,3 \\
Disfluência neurólogica & 2 & 0,9 \\
Disfagia & 14 & 6,6 \\
Discalculia & 31 & 14,6 \\
Disfonia neurológica & 5 & 2,4 \\
Agnosia visual & 1 & 0,5 \\
Normal & 1 & 0,5 \\
Agnosia auditiva & 1 & 0,5 \\
\hline
\end{tabular}

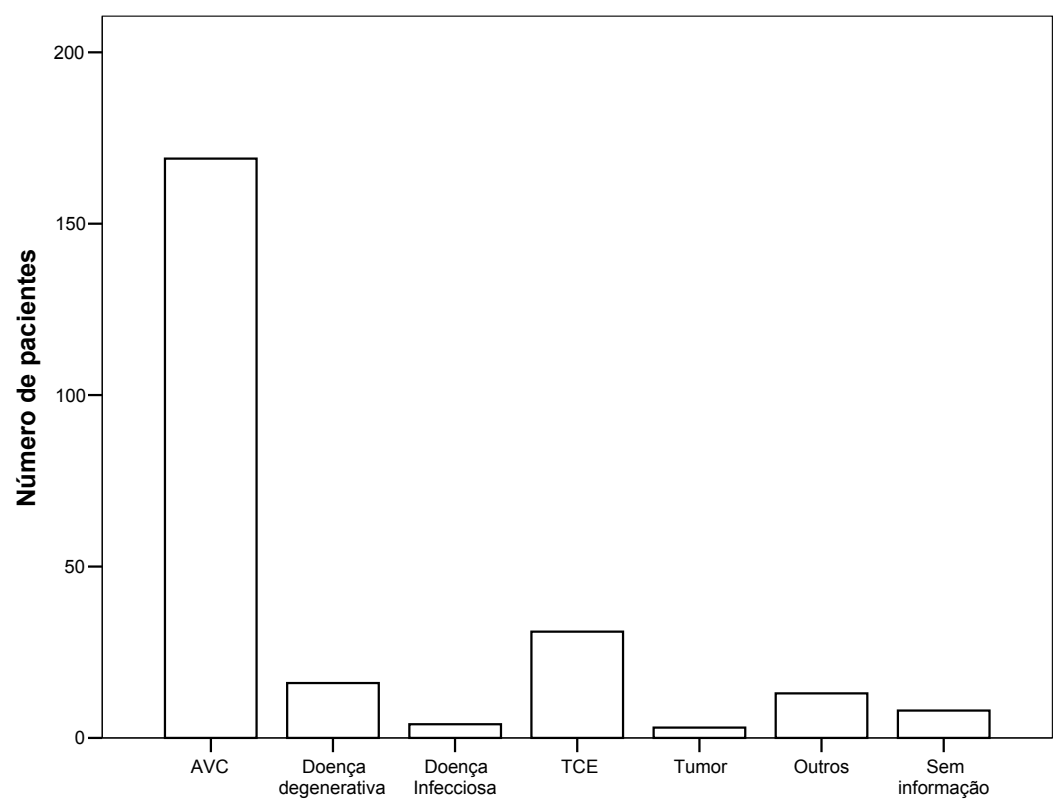

Etiologia da Lesão

Figura 4 - Distribuição do número total de pacientes em relação à etiologia da lesão.

\section{DISCUSSÃO}

Inicialmente, observamos que $56 \%$ da população atendida era do sexo masculino e $44 \%$ do sexo feminino. Em um estudo realizado no interior de SP ${ }^{9}$ também foi encontrada maior proporção de homens do que mulheres para os pacientes acometidos por AVE. Em relação especificamente aos casos de TCEs , 75,6 \% dos casos atendidos na
Rede Sarah de Hospitais do Aparelho Locomotor foram do sexo masculino. Porém a pequena diferença entre casos do sexo feminino e masculino pode ser justificada também pela idade média da população analisada no nosso estudo. Um artigo de revisão recente ${ }^{10}$ aponta que, enquanto os $A V C(s)$ predominam em homens até os 85 anos, a partir desta idade, a prevalência se inverte, sendo a população feminina mais acometida. Menopausa e idade 


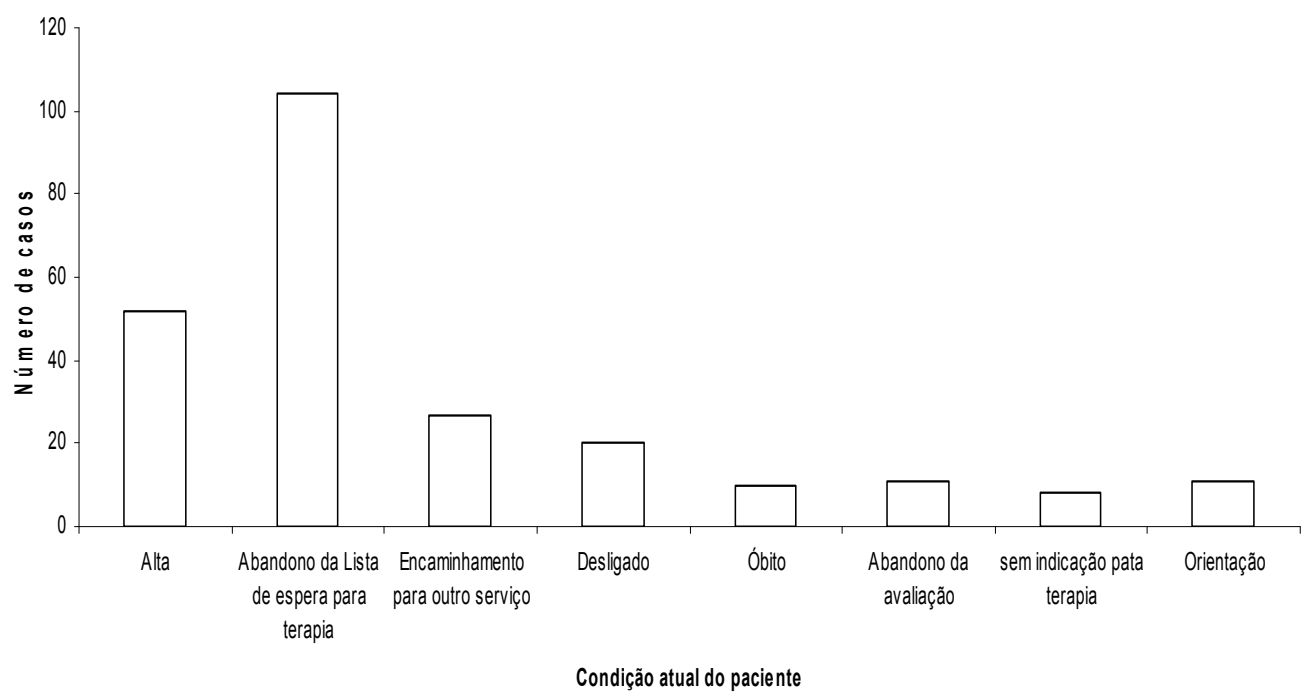

Figura 5-Distribuição do número de pacientes em relação à condição atual do paciente no ambulatório de distúrbios neurológicos adquiridos da UNIFESP (2002-2006) segundo o total de atendimentos.

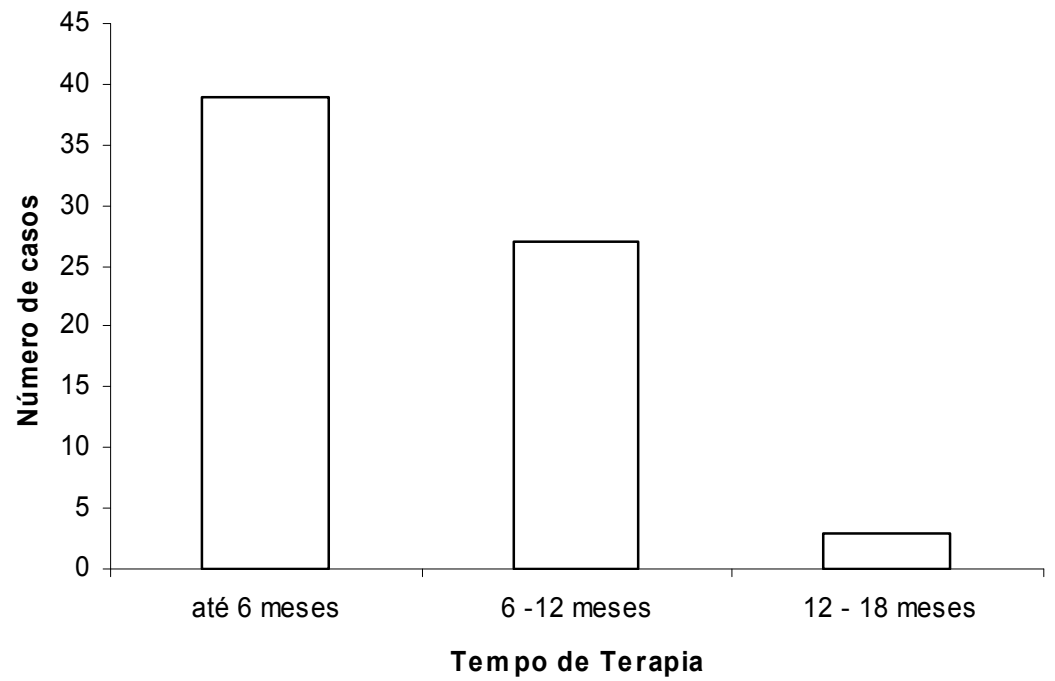

Figura 6 - Distribuição do número total de pacientes em relação ao tempo de terapia.

elevada constituíram fatores de risco biológicos associados à hipertensão e a outros fatores de risco cardiovascular, como obesidade (que é comum em mulheres com a elevação da idade) e dislipidemias ${ }^{11,12}$. Pode-se supor que com a inserção da mulher no mercado de trabalho e sua jornada dupla de trabalho, os fatores de risco para doenças cerebrovasculares aumentem para esta população e a prevalência de casos se torne semelhante ao dos homens. ${ }^{13}$ Sendo assim, a população analisada no presente apresenta características semelhantes às descritas na literatura.No entanto, existem divergências quanto ao sexo da população acometida. Algumas pesquisas retratam que o sexo masculino apresenta maior índice de lesões ${ }^{10,11,13,14}$ enquanto outros estudos evidenciam uma incidência maior no sexo feminino ${ }^{15}$

Em relação à idade, no nosso estudo, a maior parte da população $(44,3 \%)$ encontra-se entre 41 64 anos (Figura 1). Nossos resultados sugerem menor frequência de AVC, e outras etiologias cerebrovasculares, em pacientes jovens (até 20 anos de idade), possivelmente por estarem menos vulneráveis aos fatores de risco. Por outro lado, podemos encontrar idosos que muitas vezes apresentam fatores de riscos associados aumentando assim a chance de acometimento por tais etiologias $^{16-19}$. Já nas crianças, a Afasia adquirida infantil 
é considerada algo excepcional e pode ser constatada pós-lesões traumáticas, infecciosas, tumorais, associadas a doenças involutivas, entre outras ${ }^{20}$. Alguns pesquisadores observaram que a média de idade mais atingida por Acidentes Vasculares Encefálicos está em torno dos 64 anos de idade 9 . Outro estudo verificou que cerca de $10 \%$ a $20 \%$ dos casos de AVC ocorrem em indivíduos com menos de 45 anos $^{2}$. Num estudo realizado no Brasil ${ }^{20}$, em que foi feita a comparação entre a idade de pacientes afásicos por AVC e por TCE, não foi encontrada diferença estatisticamente significante. A idade média dos pacientes atendidos na Rede Sarah de Hospitais do Aparelho Locomotor com AVC foi de 60 anos. Vale ressaltar que o nosso estudo abrangeu os pacientes acometidos por outras doenças neurológicas e com diversos distúrbios da comunicação. Notamos, portanto que, com relação à faixa etária, os estudos também são bastante diversificados. Embora, existam relatos de que o risco de uma lesão vascular cerebral aumente com a idade ${ }^{18-21}$, algumas pesquisas encontram um índice maior de AVC's em populações com idade média ${ }^{2,22}$ ao passo que outros pesquisadores descreveram alta incidência de lesões vasculares em indivíduos entre 75 e 85 anos ${ }^{19,22}$.

Para melhor análise da escolaridade dos indivíduos estudados, agrupamos os pacientes em faixas que contemplassem a antiga classificação escolar: primário, ginásio, colegial e superior. Considerandose todos os casos, pudemos verificar que a maior parte dos pacientes atendidos esteve na faixa de 1 a 4 anos de estudo (Figura 2). O resultado encontrado corrobora com os achados do questionário suplementar de Pesquisa Mensal de Emprego de abril de 1996 que demonstra um predomínio populacional de adultos com 1 a 4 anos de estudo, sendo que a população de indivíduos com lesão cerebral (atendidos ambulatorialmente neste período) parece refletir o nível de escolaridade da população em geral.

Quanto aos encaminhamentos feitos para o ambulatório, em todos os anos houve predomínio do setor neurologia do HSP (Figura 3). Os encaminhamentos feitos pela rede pública de saúde ocorrem muitas vezes para o ambulatório de especialidades que é de referência para este tipo de atendimento. Outra justificativa cabível seria o conhecimento do serviço por vários profissionais da rede de saúde em geral. De fato, podemos destacar a importância desses encaminhamentos, já que alguns autores observaram que $25 \%$ dos pacientes atendidos com AVE apresentavam alteração de fala ou linguagem ${ }^{23}$. Entre as manifestações tardias para o AVC podemos encontrar: déficits motores, incapacidade funcional, distúrbios de linguagem, disfagia, depressão, desajuste social e piora na qualidade de vida $^{2}$. A literatura compilada corrobora aos resultados encontrados e sustenta a importância da relação dos neurologistas com esta especialidade da fonoaudiologia ${ }^{4,7}$.

Em relação aos diagnósticos fonoaudiológicos, encontramos os seguintes resultados: $56,1 \%$ de diagnósticos de afasia, 33\% de disartria, 19,3\% de apraxia, $14,6 \%$ de discalculia, $11,8 \%$ de distúrbio sutil de linguagem, $9,4 \%$ de distúrbio linguísticocognitivo, $6,6 \%$ de disfagia, $2,8 \%$ de agrafia adquirida, 2,4\% de disfonia neurológica, 1,9\% de distúrbio de aquisição de fala e linguagem, 0,9\% de dislexia adquirida e $0,5 \%$ de agnosia visual e $0,5 \%$ agnosia auditiva (Tabela 1). Classicamente, temos as afasias, as dislexias e as disgrafias como distúrbios de linguagem causados por lesões no SNC4. Sobre as condições etiológicas da apraxia, estas incluem acidentes vasculares cerebrais, doenças degenerativas, traumas e tumores, desde que as lesões centrais se localizem em áreas responsáveis pela sequencialização dos comandos motores da fala7. As Disartrias são causadas por lesões decorrentes do sistema nervoso central ou periférico, que provocam problemas na comunicação oral devido a uma paralisia, fraqueza ou incoordenação da musculatura da fala ${ }^{24}$. Perfis de pacientes disártricos já foram descritos em nossa população ${ }^{25}$. $\mathrm{Em}$ relação aos pacientes com lesão do SNC, observamos, neste estudo, que houve um predomínio de distúrbios de linguagem em relação aos distúrbios da fala. A mesma tendência ocorreu na análise de distúrbios da comunicação numa população genérica ${ }^{26}$. No entanto, no referido estudo, ao analisarem-se os dados relativos aos distúrbios da comunicação pós- AVE em adultos, a incidência de casos de disartria e de afasia se equipararam. Ressalta-se, no entanto, que a casuística oriunda de um ambulatório especializado, provavelmente seja mais fidedigna do que ocorre na população em geral.

Em relação à frequência das etiologias dos diagnósticos fonoaudiológicos em todos os anos, observamos que a maior parte $(69,4 \%)$ dos pacientes foram acometidos por o Acidente Vascular Cerebral (Figura 4). Estudos revelam que a doença cérebro vascular é causa mais frequente de morbidades e óbitos de pacientes, no Município de São Paulo ${ }^{3,8}$. No Brasil, o AVC é atualmente considerado a primeira causa de morte em adultos ${ }^{2}$. Já o TCE é importante causa de morte, sendo superado apenas pelo AVC e, quando há sobrevida normalmente ocorrem alterações físicas, psíquicas e emocionais, $\mathrm{Na}$ rede Sarah, em 2001, foram atendidos 344 pacientes com TCE e 1419 pacientes com AVC. ${ }^{20}$ 
Como podemos observar os dados encontrados corroboram com os descritos na literatura.

Em relação à análise da condição do paciente no serviço terciário pudemos observar, na Figura 5, pacientes que desistiram da vaga para terapia, pacientes que tiveram alta da terapia, os que foram encaminhados para outros serviços, os que foram desligados da terapia, houve abandono da avaliação, os que não foram candidatos para reabilitação, os que apenas receberam orientação e aqueles que foram a óbito (dados apresentados em ordem decrescente de frequência). Os casos mais prevalentes foram dos pacientes atendidos em avaliação inicial que acabaram desistindo da vaga para a terapia. Como justificativa para este número, tivemos fatores como: pacientes que encontraram vaga de terapia próxima à residência, que estavam fazendo algum tipo de tratamento e não apresentaram disponibilidade para comparecer ao serviço, agravo da doença, mudança de cidade, apresentação de melhora espontânea e a grande maioria dos casos estavam entre os pacientes que não puderam ser contatados. A segunda condição foi o número de casos atendidos na avaliação inicial que fizeram terapia, atingiram os objetivos propostos para sua reabilitação e receberam alta, ou atingiram o limite terapêutico (pacientes que não apresentam mais evolução em terapia). Em alguns casos, o paciente foi encaminhado para outro serviço ou porque apresentou necessidade de uma equipe multiprofissional para atender melhor as suas sequelas ou teve dificuldade de aderência à terapia (pacientes que moram longe, por exemplo). Os pacientes desligados do nosso serviço são aqueles que faltaram sem justificativa por três vezes consecutivas ou 5 alternadas, apenas um caso foi desligado por limite terapêutico, pois o paciente não aceitava a alta. Os pacientes que não foram candidatos a terapia e os que foram orientados em sua maioria são pacientes que foram triados e não apresentaram necessidade de reabilitação por serem casos leves ou normais. Estes são basicamente casos que tiveram uma melhora espontânea, no período entre o encaminhamento e a convocação para o atendimento. De fato, o período da recuperação espontânea é bastante discutido na literatura ${ }^{27}$, sendo que alguns autores acreditam que as mudanças possam naturalmente ocorrer devido à participação do hemisfério direito na recuperação ${ }^{28}$. Os pacientes que foram a óbito durante o período de lista de espera ou durante a terapia também foram contabilizados.

Em relação ao tempo de terapia, na Figura 6, verificamos que a maioria dos pacientes apresentou alta até os 6 primeiros meses de terapia, seguido pelos que necessitam de12 meses e por fim os que realizam até 18 meses. Quanto ao tempo de terapia, os fatores realmente relevantes são a gravidade inicial do problema (que está intimamente ligada à extensão e à localização da lesão) determinantes do prognóstico ${ }^{29}$ e por sua vez, justificando o número de meses necessários para se obter alta. Segundo a Rede Sarah de Hospitais do Aparelho Locomotor, os primeiros três a seis meses após o AVC são os mais importantes no processo de readaptação. A linguagem, o equilíbrio e as habilidades funcionais podem continuar melhorando até dois anos após a lesão.

\section{Implicações do estudo}

Trata-se de um estudo inicial em que objetivouse verificar o perfil populacional dos pacientes com distúrbios da comunicação de origem neurológica $O$ cenário deste estudo foi um Hospital escola situado na cidade de São Paulo que atende a uma grande demanda populacional, portanto, os dados obtidos merecem algumas considerações. As etiologias mais comuns em nosso meio para estes distúrbios seguiram as observadas no mundo todo, tanto em países em desenvolvimento quanto em países desenvolvidos: o AVC e o TCE- este último sobretudo por acidentes automobilísticos. Portanto, este estudo sugere que grande parte dos casos acometidos por este distúrbio poderiam ter sido evitados com campanhas de educação para o trânsito e de prevenção dos fatores de risco para o AVC. Além disso, perfis populacionais de um serviço de referência podem colaborar para obtenção de dados epidemiológicos mais abrangentes bem como para re-estruturar o sistema de atendimento à população.

\section{CONCLUSÃO}

A partir dos dados obtidos foi possível concluir que:

1. A Afasia foi o transtorno de linguagem de maior prevalência entre número total de casos atendidos; o mesmo ocorreu com a disartria em relação à fala;

2. O Acidente Vascular Cerebral foi a etiologia neurológica que prevaleceu entre número total de casos atendidos;

3. A análise ano a ano revelou os mesmos dados gerais, ou seja; com relação aos distúrbios da comunicação, a afasia e a disartria foram os mais frequentes diagnósticos fonoaudiológicos e o acidente vascular cerebral se destacou como a etiologia que mais acometeu os pacientes; 
4. $50 \%$ dos casos puderam ser atendidos em programas de reabilitação em relação ao número geral de casos atendidos;

5. A maioria dos pacientes foram encaminhados pelo setor de Neurologia do Hospital São Paulo - UNIFESP.

\section{AGRADECIMENTOS}

Agradecemos a Fundação de Amparo à Pesquisa do Estado de São Paulo pelo financiamento concedido a este estudo.

\section{ABSTRACT}

Purpose: to characterize the population of patients with neurological lesion with communication disorders, assisted by a Tertiary Hospital. Specific objectives 0 to check the prevalence of each type of speech and / or language disorder, neurological etiologies and the origin of the referrals for the total of the patients. Method: we analyzed all medical records of seen patients in the past 5 years (2002 to 2006) from the outpatient clinic of Acquired Neurological Disorders - Department of Speech Pathology and Audiology (UNIFESP) through a retrospective study. Result: the study showed 244 cases in the period from 2002 to 2006 . As for the characterization of the sample, the following data were found, namely: for gender, $56 \%$ were men; $44.3 \%$ aged from 41 to 64 years; for schooling, $39.8 \%$ of the patients had 1 to 4 year schooling; for the referrals $35.7 \%$ were from the Neurology outpatient clinic of UNIFESP; for the injury etiology $69.3 \%$ were diagnosed with stroke. Aphasia and dysartrhias were the most common disorders. Conclusion: the stroke and traumatic brain injury stood out as the etiology that most attached the patients; In relation to the overall number of evaluated cases, it was found that $50 \%$ of the group have had rehabilitation program and the majority of the patients were referred from the service of Neurology, Hospital São Paulo - UNIFESP.

Keywords: Communication Disorders; Brain Injuries; Epidemiology

\section{REFERÊNCIAS BIBLIOGRÁFICAS}

1. Engelter ST, Gostynski M, Papa S, Frei M, Born C, Ajdacic-Gross V, Gutzwiller F, Lyrer PA. Epidemiology of aphasia attributable to first ischemic stroke: incidence, severity, fluency, etiology, and thrombolysis. Strokes, Switzerland, Jun. 2006; 37 (6): 1379-84.

2. Fukujima MM. Acidente VascularCerebral. In: Ortiz KZ. Distúrbios Neurológicos Adquiridos:Linguagem e Cognição, 2a ed , Baurueri, SP: Manole, 2010, p.34-44.

3. Oliveira C O de; Ikuta N, Regner, A. Biomarcadores prognósticos no traumatismo crânio-encefálico grave. Rev. bras. ter. intensiva [online]. 2008, 20(4): 411-21.

4. Ortiz KZ. Distúrbios Neurológicos Adquiridos:Linguagem e Cognição, $2^{\underline{a}}$ ed, Baurueri, SP: Manole, 2010, 484p.

5. Beal C. Gender and stroke symptoms: a review of the current literature. J. neurosci Nurs, 2010, 42(2): 80-7.

6. Spencer KA, Slocomb DL. The neural basis of ataxic dysarthria. Cerebellum, USA, 2007; 6 (1):58-65.
7. Ortiz KZ. Distúrbios Neurológicos Adquiridos: Fala e Deglutição, $2^{\text {a }}$ ed, Baurueri, SP: Manole, 2010, 387p.

8. Mansur LL, Radanovic M. Neurolinguística: Princípios para clínica, São Paulo, SP: El - Edições Inteligentes, 2004.

9. Rodrigues JE, Sá M da S; Alouche S. Perfil dos pacientes acometidos por AVE tratados na clínica escola de fisioterapia da UMESP. Revista Neurociências.Brasil, 2004; (12) 3: 117-22.

10. Persky RW, Turtzo LC, McCullough LD. Stroke in women: disparities and outcomes. Curr Cardiol Rep, 2010, 12(1):6-13.

11. Lessa I, Magalhães L, Araújo MJ, Almeida Filho $\mathrm{N}$, Aquino E, Oliveira MMC Hipertensão arterial na população adulta de Salvador (BA) - Brasil. Arquivos Brasileiros de Cardiologia, 2006; (87) : 6

12. Jamieson DG, SKliut $M$. stroke in Women: What is Different? Curr Atheroscler Rep 2010, epub ahead of print.

13. Ferris A, Robertson RM, Fabunmi R, Mosca L. American Heart Association and American Stroke Association national survey of stroke risk awareness among women. Circulation. 2005; 111(10): 1321-6. 
14. Lofmark U, Hammarstrom A. Evidence for Age-Dependent Education-Related Differences in Men and Women with First-Ever Stroke. Results from a Community-Based Incidence Study in Northern Sweden. Neuroepidemiology. 2007; 28(3):135-41.

15. Appelros P, Stegmayr B, Terent A. Sex Differences in Stroke Epidemiology: A Systematic Review. Stroke. 2009; 40:1082.

16. Dulli D, Samaniego EA. Inpatient and community ischemic strokes in a university hospital. Neuroepidemiology. 2007; 28 (2): 86-92.

17. Crimmins EM, Kim JK,Sole-Auró A. Gender Differences in Hrealth: results from SHARE, ELSA and HRS. The European Journal of Public Health. Doi:10.1093/eurpub/ckq022. 2010

18. Frost L, Vukelic Andersen L, Godtfredsen $\mathrm{J}$, Mortensen LS. Age and risk of stroke in atrial fibrillation: evidence for guidelines? Neuroepidemiology. 2007; 28(2):109-15.

19. Plittella JEH, Duarte JE. Prevalência e padrão de distribuição das doenças cerebrovasculares em 242 idosos, procedentes de um hospital geral, necropsiados em Belo Horizonte, Minas Gerais, no período de 1976 a 1997. Arq. Neuro-Psiquiatr., 2002(60) 1: 47-55.

20. Oliveira KT, Moura-Ribeiro MVL, Ciasca SM. Doença cerebrovascular: aquisição de linguagem em pré-escolares Arq. Neuro-Psiquiatr. São Paulo, 2005: (63) 3b.

21. Centers for Disease Control and Prevention (CDC). Prevalence of stroke--United States, 2005. MMWR Morb Mortal Wkly Rep. 2007; 56(19):469-74.
22. Arnold M, Kurmann R, Galimanis A, Sarikaya H, Stapf C, Gralla J, Georgiadis D, Fisher U, Mattle HP, Bousser M, Baumgartner, RW. Differences in Demographic Characteristics and Risk Factors in patients with spontaneous vertebral artery dissections with and without ischemic events. Stroke, 2010: 41: 802-4.

23. Rede Sarah de Hospitais de Reabilitação. Acessado em: 16/06/2008. Disponível em: www. sarah.br.

24. Darley FL, Aronson AE, Brown JR. Clusters of diagnostic patterns of dysarthria. Journal Speech and Hearing Research 1969;12: 249-61.

25. Fornachari A, Ortiz KZ.Perfil populacional de pacientes com Disartria atendidos em hospital terciário.Rev Soc Brás Fonoaudiol 2009; 14(3): 446-53.

26. Boacnin, SBO. Desordens da comunicaçäo humana: perfil populacional. Tese. São Paulo, SP: Universidade Federal de Säo Paulo - Escola Paulista de Medicina. 2001.

27. Cappa SF. Neuroimaging of recovery from aphasia. Neuropsychological Rehabilitation, 2000, 10(3), 365-76.

28. Breier JI, Juranek J, Maher LM, Schmadeke S, Men D, Papanicolaou AC. Behavioral and neurophysiologic response to therapy for chronic aphasia. Arch Phys Med Rehabil. 2009;90(12):2026-33.

29. Basso, A. Fatores de Prognósticos em Afasia. In: Mansur, LL E Rodrigues, N. Temas em Neurolinguística, SP: Tec Art, 1993,63.

DOI: 10.1590/S1516-18462010005000097

RECEBIDO EM: 24/02/2010

ACEITO EM: 21/06/2010

Endereço para correpondência:

Karin Zazo Ortiz

Rua Cunha, 111, conjunto 73, Vila Clementino

São Paulo - SP - Brasil

CEP: 04037-030

E-mail: karinortiz.fono@epm.br 